\title{
53 Endurance training and blood pressure
}

Blood pressure results from the regulated interplay between the heart and the blood vessels. It fluctuates significantly, with higher readings during physical activity than at rest, and is higher during the day than at night. Blood pressure consists of two phases: the systole, when the heart muscle contracts, and the diastole, when the heart muscle relaxes. Arterial hypertension occurs when the blood pressure in the arterial system is permanently elevated. in lay terms, it is simply called high blood pressure. According to the $\mathrm{WHO}$, arterial hypertension is defined as a systolic blood pressure equal to or above $140 \mathrm{mmHg}$ and/or a diastolic pressure equal to or above $90 \mathrm{mmHg}$. The results of a Swedish study of 1.2 million men, observed over 24 years, suggest that a higher diastolic pressure poses an increased risk in young adults, whereas the systolic blood pressure is more relevant in older individuals (Sundström et al. 2011).

The lack of early warning symptoms is the really confounding part about this disease. High blood pressure often remains undiagnosed for years and causes significant, irreversible damage over time. This includes, among other things, vascular damage with the possible development of coronary heart disease, myocardial infarction, stroke, ocular and renal damage or dementia. Nearly half of all deaths in under-65-year-olds are associated with hypertension-related diseases (•Tab. 53.1).

Blood pressure is regulated by hormones via the renin-angiotensin-aldosterone system. About 30\% of the adult population in Germany is permanently affected by hypertension, and more than 1 in 5 adults worldwide. Early diagnosis in young adults would be possible and useful, but seldom happens (Allen

\begin{tabular}{|l|l|l|}
\hline & $\begin{array}{l}|c| \\
\text { Systolic } \\
(\mathrm{mmHg})\end{array}$ & $\begin{array}{l}\text { Diastolic } \\
(\mathrm{mmHg})\end{array}$ \\
\hline Optimal blood pressure & $<120$ & $<80$ \\
\hline Normal blood pressure & $120-129$ & $80-84$ \\
\hline High normal blood pressure & $130-139$ & $85-89$ \\
\hline Prehypertension) & $140-159$ & $90-99$ \\
\hline Stage 1 hypertension & $160-179$ & $100-109$ \\
\hline Stage 2 hypertension & $>180$ & $>110$ \\
\hline $\begin{array}{l}\text { Stage } 3 \text { hypertension } \\
\text { (hypertensive emergency) }\end{array}$ & & \\
\hline Isolated systolic hypertension & $>140$ & $<90$ \\
\hline
\end{tabular}

et al. 2014). High blood pressure is treated with medication, although it is very important that those afflicted change their lifestyle as well. This includes weight reduction, increasing the consumption of fruit and vegetables, cooking with unsaturated fats, reducing salt intake, restricting sugary drinks, giving up smoking, restricting alcohol intake and avoiding long-term stress (Aburto et al. 2013, Mancia et al. 2013, Yokoyama et al. 2014).

Physical exercise is a paramount component of basic therapy. It is mainly endurance training that promote the cardiovascular system. Regular exercise leads to a long-term reduction in systolic values by about $10-15 \mathrm{mmHg}$ and in diastolic values by about $5-10 \mathrm{mmHg}$ ( $\triangleright$ Chapter 49 to $\triangleright$ Chapter 51 ). The involvement of a sports physician should be compulsory. 